\title{
Weighted Endpoint Estimates for Commutators of Riesz Transforms Associated with Schrödinger Operators
}

\author{
Yu Liu, Jielai Sheng, and Lijuan Wang \\ School of Mathematics and Physics, University of Science and Technology Beijing, Beijing 100083, China \\ Correspondence should be addressed to Yu Liu; liuyu75@pku.org.cn
}

Received 14 May 2013; Accepted 1 October 2013

Academic Editor: Baoxiang Wang

Copyright ( 2013 Yu Liu et al. This is an open access article distributed under the Creative Commons Attribution License, which permits unrestricted use, distribution, and reproduction in any medium, provided the original work is properly cited.

Let $L=-\Delta+V$ be a Schrödinger operator, where $\Delta$ is the laplacian on $\mathbb{R}^{n}$ and the nonnegative potential $V$ belongs to the reverse Hölder class $B_{s_{1}}$ for some $s_{1} \geq(n / 2)$. Assume that $\omega \in A_{1}\left(\mathbb{R}^{n}\right)$. Denote by $H_{L}^{1}(\omega)$ the weighted Hardy space related to the Schrödinger operator $L=-\Delta+V$. Let $\mathscr{R}_{b}=[b, \mathscr{R}]$ be the commutator generated by a function $b \in \mathrm{BMO}_{\theta}\left(\mathbb{R}^{n}\right)$ and the Riesz transform $\mathscr{R}=\nabla(-\Delta+V)^{-(1 / 2)}$. Firstly, we show that the operator $\mathscr{R}$ is bounded from $L^{1}(\omega)$ into $L_{\text {weak }}^{1}(\omega)$. Secondly, we obtain the endpoint estimates for the commutator $[b, \mathscr{R}]$. Namely, it is bounded from the weighted Hardy space $H_{L}^{1}(\omega)$ into $L_{\text {weak }}^{1}(\omega)$.

\section{Introduction}

Let $L=-\Delta+V$ be a Schrödinger operator, where $\Delta$ is the laplacian on $\mathbb{R}^{n}$ and the nonnegative potential $V$ belongs to the reverse Holder class $B_{s_{1}}$ for some $s_{1} \geq(n / 2)$ and $n \geq 3$. In this paper, we consider the Riesz transform associated with the Schrödinger operator $L$ as follows:

$$
\mathscr{R}=\nabla(-\Delta+V)^{-1 / 2} .
$$

Let $b$ be a locally integrable function on $\mathbb{R}^{n}$ and let $T$ be a linear operator. For a suitable function $f$, the commutator is defined by $T_{b} f \doteq[b, T] f=b T(f)-T(b f)$. It is well known that when $T$ is a Calderón-Zygmund operator, Coifman et al. $[1]$ proved that $[b, T]$ is a bounded operator on $L^{p}$ for $1<p<$ $\infty$ if and only if $b \in \operatorname{BMO}\left(\mathbb{R}^{n}\right)$.

Recently, some scholars have investigated the boundedness of the commutators generated by a BMO function $b$ and Riesz transforms associated with the Schrödinger operator (cf. [2-8]). It follows from [9] that Riesz transform associated with the Schrödinger operator $L$ is not a Calderón-Zygmund operator if the potential $V \in B_{s}((n / 2)<s<n)$. Their results imply that the boundedness of the commutators of Riesz transform associated with the Schrödinger operator $L$ depends on the nonnegative potential $V$. In [5], the authors have obtained the weighted $L^{p}, 1<p<\infty$, and weak $L \log L$ the estimates for the commutator $\mathscr{R}_{b}$. In this paper we are interested in the weighted Hardy space estimates for $\mathscr{R}_{b}$, which are also the weighted endpoint estimates. It is noted that our main results generalize Theorem 2.7 and Theorem 4.1 in [3] to the weighted case and the function $b$ that we consider belongs to a larger class than the classical BMO space.

Note that a nonnegative locally $L^{s}$ integrable function $V$ on $\mathbb{R}^{n}$ is said to belong to $B_{s}(1<s<\infty)$ if there exists $C>0$ such that the reverse Hölder inequality

$$
\left(\frac{1}{|B|} \int_{B} V(x)^{s} d x\right)^{1 / s} \leq \frac{C}{|B|} \int_{B} V(x) d x
$$

holds for every ball $B$ in $\mathbb{R}^{n}$. Obviously, $B_{s_{2}} \subset B_{s_{1}}$ if $s_{2}>s_{1}$. But it is important that the $B_{q}$ class has a property of "selfimprovement"; that is, if $V \in B_{s}$, then $V \in B_{s+\varepsilon}$ for some $\varepsilon>0$.

Assume $V \in B_{s_{1}}$ for some $s_{1}>(n / 2)$. Then the auxiliary function $\rho(x, V)=\rho(x)$ introduced by Shen in [9] is defined as follows:

$$
\begin{aligned}
\rho(x) & =\frac{1}{m(x, V)} \\
& \doteq \sup _{r>0}\left\{r: \frac{1}{r^{n-2}} \int_{B(x, r)} V(y) d y \leq 1\right\}, \quad x \in \mathbb{R}^{n} .
\end{aligned}
$$


In [4], the authors define the class $\mathrm{BMO}_{\theta}(\rho)$ of locally integrable function $b$ such that

$$
\frac{1}{|B(x, r)|} \int_{B(x, r)}\left|b(y)-b_{B}\right| d y \leq C\left(1+\frac{r}{\rho(x)}\right)^{\theta},
$$

for all $x \in \mathbb{R}^{n}$ and $r>0$, where $\theta>0$ and $b_{B}=$ $(1 /|B|) \int_{B} b(y) d y$. A norm for $b \in \mathrm{BMO}_{\theta}(\rho)$, denoted by $[b]_{\theta}$, is given by the infimum of the constants satisfying (4), after identifying functions that differ upon a constant. If we let $\theta=0$ in (4), then $\operatorname{BMO}_{\theta}(\rho)$ is exactly the John-Nirenberg space BMO. Denote $\mathrm{BMO}_{\infty}(\rho)=\bigcup_{\theta>0} \mathrm{BMO}_{\theta}(\rho)$. It is easy to see that $\mathrm{BMO} \subset \mathrm{BMO}_{\theta}(\rho) \subset \mathrm{BMO}_{\theta^{\prime}}(\rho)$ for $0<\theta \leq \theta^{\prime}$. Hence, $\mathrm{BMO} \subset \mathrm{BMO}_{\infty}(\rho)$.

Throughout this paper, we set $\omega(E)=\int_{E} \omega(x) d x$ for any subset $E \subseteq \mathbb{R}^{n}$. Assume that the nonnegative function $\omega \in$ $L_{\text {loc }}^{1}\left(\mathbb{R}^{n}\right)$. We say that $\omega \in A_{p}\left(\mathbb{R}^{n}\right)$ for $1<p<\infty$ if there is a constant $C>0$ such that

$$
\sup _{B}\left(\frac{1}{|B|} \int_{B} \omega(x) d x\right)\left(\frac{1}{|B|} \int_{B}(\omega(x))^{1-p^{\prime}} d x\right)^{p-1} \leq C,
$$

for all balls $B$ in $\mathbb{R}^{n}$, where $(1 / p)+\left(1 / p^{\prime}\right)=1$.

We say that $\omega \in A_{1}\left(\mathbb{R}^{n}\right)$, if there exists a positive constant $C>0$, such that

$$
M \omega(x) \leq C \omega(x), \quad \text { a.e. } x \in \mathbb{R}^{n},
$$

where $M$ is a Hardy-Littlewood operator.

Given a weight function $\omega \in A_{p}\left(\mathbb{R}^{n}\right)$ for $1 \leq p<$ $\infty$, as usual we denote by $L^{q}(\omega)$ the space of all measurable functions satisfying

$$
\|f\|_{L^{q}(\omega)}^{q}=\int_{\mathbb{R}^{n}}|f(x)|^{q} \omega(x) d x<\infty .
$$

When $q=\infty, L^{\infty}(\omega)$ will be taken to mean $L^{\infty}$ and $\|f\|_{L^{\infty}(\omega)}=\|f\|_{L^{\infty}}$. Moreover, denote by $L_{\text {weak }}^{1}(\omega)$ the space of all measurable functions satisfying

$$
\sup _{\lambda>0}\left[\lambda \omega\left\{x \in \mathbb{R}^{n}:|f(x)| \geq \lambda\right\}\right]<\infty .
$$

In the rest of this paper, we always assume that $\omega \epsilon$ $A_{1}\left(\mathbb{R}^{n}\right)$.

Because $V \geq 0$ and $V \in L_{\text {loc }}^{n / 2}\left(\mathbb{R}^{n}\right)$, the Schrödinger operator $L$ generates a $\left(C_{0}\right)$ contraction semigroup $\left\{T_{s}^{L}: s>\right.$ $0\}=\left\{e^{-s L}: s>0\right\}$. The maximal function associated with $\left\{T_{s}^{L}: s>0\right\}$ is defined by $M^{L} f(x)=\sup _{s>0}\left|T_{s}^{L} f(x)\right|$.

The Hardy space $H_{L}^{1}\left(\mathbb{R}^{n}\right)$ associated with the Schrödinger operator $L$ is defined in terms of the maximal function mentioned above (cf. [10]). Recently, the weighted Hardy space $H_{L}^{1}(\omega)$ has been established by Liu et al. [11] and it is defined as follows.

Definition 1. A function $f \in L^{1}(\omega)$ is said to be in $H_{L}^{1}(\omega)$ if the maximal function $M^{L} f$ belongs to $L^{1}(\omega)$. The norm of such a function is defined by $\|f\|_{H_{L}^{1}(\omega)}=\left\|M^{L} f\right\|_{L^{1}(\omega)}$.
Definition 2. Let $1 \leq q \leq \infty$. A function $a \in L^{q}(\omega)$ is called an $H_{L}^{1, q}(\omega)$-atom if $r<\rho\left(x_{0}\right)$ and the following conditions hold:

(i) $\operatorname{supp} a \subset B\left(x_{0}, r\right)$,

(ii) $\|a\|_{L^{q}(\omega)} \leq \omega\left(B\left(x_{0}, r\right)\right)^{(1 / q)-1}$,

(iii) if $r<\left(\rho\left(x_{0}\right) / 4\right)$, then $\int_{B\left(x_{0}, r\right)} a(x) d x=0$.

In [11], Liu et al. gave the following atomic decomposition for the space $H_{L}^{1}(\omega)$.

Proposition 3. Assume $V \in B_{s_{1}}$ for some $s_{1}>(n / 2)$. Let $f \in$ $L^{1}(\omega)$. Then $f \in H_{L}^{1}(\omega)$ if and only if $f$ can be written as $f=$ $\sum_{j} \lambda_{j} a_{j}$, where $a_{j}$ are $H_{L}^{1, \infty}(\omega)$-atoms, $\sum_{j}\left|\lambda_{j}\right|<\infty$, and the sum converges in the $H_{L}^{1}(\omega)$ quasinorm. Moreover,

$$
\|f\|_{H_{L}^{1}(\omega)} \sim \inf \left\{\sum_{j}\left|\lambda_{j}\right|\right\},
$$

where the infimum is taken over all atomic decompositions of $f$ into $H_{L}^{1, \infty}$-atoms.

Following the above definition of atoms and the above atomic decomposition, we know that the weighted Hardy space $H_{L}^{1}(\omega)$ is not the special case of Hardy spaces established by Yang and Zhou in [12].

Now we are in a position to give the main results in this paper.

Theorem 4. Let $\omega \in A_{1}\left(\mathbb{R}^{n}\right)$. Suppose $V \in B_{s}$ for $s>(n / 2)$. Then,

$$
\begin{array}{r}
\omega\left\{x \in \mathbb{R}^{n}:\left|\nabla(-\Delta+V)^{1 / 2} f(x)\right|>\alpha\right\} \leq \frac{C}{\alpha}\|f\|_{L^{1}(\omega)}, \\
\text { for every } \alpha>0 .
\end{array}
$$

Theorem 5. Let $b \in B M O_{\infty}(\rho)$. Then, for any $\lambda>0$,

$$
\begin{array}{r}
\omega\left(x \in \mathbb{R}^{n}:\left|\mathscr{R}_{b} f(x)\right|>\lambda\right) \leq \\
\quad \frac{C[b]_{\theta}}{\lambda}\|f\|_{H_{L}^{1}(\omega)}, \\
\forall f \in H_{L}^{1}(\omega),
\end{array}
$$

where $\theta>0$. Namely, the commutator $\mathscr{R}_{b}$ is bounded from $H_{L}^{1}(\omega)$ into $L_{\text {weak }}^{1}(\omega)$.

This paper is organized as follows. In Section 2, we recall some basic facts to prove main results in this paper. Section 3 gives the proof of weighted estimates of Riesz transform associated with the Schrödinger operator. In Section 4, we prove Theorem 5.

Throughout this paper, the letter $C$ stands for a constant and is not necessarily the same at each occurrence. By $B_{1} \sim B_{2}$, we mean that there exists a constant $C>1$ such that $(1 / C) \leq$ $\left(B_{1} / B_{2}\right) \leq C$. Moreover, for the ball $B=B(x, r)$, we denote the ball $M B$ by $M B=B(x, M r)$, where $M$ is a positive constant. 


\section{Preliminaries}

Firstly, we recall some lemmas of the auxiliary function $\rho(x)$ which have been proved by Shen in [9]. Throughout this section we always assume $V \in B_{s_{1}}$ for some $s_{1}>(n / 2)$.

Lemma 6. The measure $V(x) d x$ satisfies the doubling condition; that is, there exists $C>0$ such that

$$
\int_{B(x, 2 r)} V(y) d y \leq C \int_{B(x, r)} V(y) d y
$$

holds for all balls $B(x, r)$ in $\mathbb{R}^{n}$.

Lemma 7. For $0<r<R<\infty$,

$$
\begin{aligned}
1 / r^{n-2} & \int_{B(x, r)} V(y) d y \\
& \leq C\left(\frac{R}{r}\right)^{\left(n / s_{1}\right)-2} 1 / R^{n-2} \int_{B(x, R)} V(y) d y .
\end{aligned}
$$

Lemma 8. If $r=\rho(x)$, then

$$
1 / r^{n-2} \int_{B(x, r)} V(y) d y=1 .
$$

Moreover,

$$
1 / r^{n-2} \int_{B(x, r)} V(y) d y \sim 1 \quad \text { if and only if } r \sim \rho(x) .
$$

Lemma 9. There exists $l_{0}>0$ such that, for any $x$ and $y$ in $\mathbb{R}^{n}$,

$$
\frac{1}{C}\left(1+\frac{|x-y|}{\rho(x)}\right)^{-l_{0}} \leq \frac{\rho(y)}{\rho(x)} \leq C\left(1+\frac{|x-y|}{\rho(x)}\right)^{l_{0} /\left(l_{0}+1\right)} .
$$

In particular, $\rho(x) \sim \rho(y)$ if $|x-y|<C \rho(x)$.

Lemma 10. There exists $l_{1}>0$ such that

$$
\begin{aligned}
& \int_{B(x, R)} \frac{V(y)}{|x-y|^{n-2}} d y \\
& \quad \leq \frac{C}{R^{n-2}} \int_{B(x, R)} V(y) d y \leq C\left(1+\frac{R}{\rho(x)}\right)^{l_{1}} .
\end{aligned}
$$
to [9].

For the proofs of Lemma 6 to Lemma 10, readers can refer

A ball $B(x, \rho(x))$ is called critical. Due to Lemma 2.3 in [10], we have Lemma 12 on $\mathbb{R}^{n}$.

Proposition 11. There exists a sequence of points $\left\{x_{k}\right\}_{k=1}^{\infty}$ in $\mathbb{R}^{n}$, such that the family of critical balls $Q_{k}=B\left(x_{k}, \rho\left(x_{k}\right)\right), k \geq 1$, satisfies the following

(i) $\bigcup_{k} Q_{k}=\mathbb{R}^{n}$.

(ii) There exists $N=N(\rho)$ such that, for every $k \in \mathbb{N}$, card $\left\{j: 4 Q_{j} \cap 4 Q_{k} \neq \emptyset\right\} \leq N$.
Secondly, we recall the estimates of the fundamental solution of the operator $-\Delta+V+i \tau$ and give the estimates of kernels of Riesz transform $\mathscr{R}$. Let $\Gamma(x, y, \tau)$ denote the fundamental solution for the operator $-\Delta+V+i \tau$, where $\tau \in \mathbb{R}$. Clearly, $\Gamma(x, y, \tau)=\Gamma(y, x,-\tau)$.

Lemma 12. Let $N>0$ be an integer.

(1) Suppose $V \in B_{s_{1}}$ for $s_{1}>(n / 2)$. Then there exists $C_{N}>$ 0 such that, for $x \neq y$,

$$
\begin{aligned}
& |\Gamma(x, y, \tau)| \\
& \quad \leq \frac{C_{N}}{\left(1+|x-y||\tau|^{(1 / 2)}\right)^{N}\left(1+|x-y| \rho(x)^{-1}\right)^{N}} \frac{1}{|x-y|^{n-2}} .
\end{aligned}
$$

(2) Suppose $V \in B_{s_{1}}$ for $s_{1}>(n / 2)$. Then there exists $C_{N}>$ 0 such that, for $x \neq y$,

$$
\begin{aligned}
\left|\nabla_{x} \Gamma(x, y, \tau)\right| & \leq \frac{C_{N}}{\left(1+|x-y||\tau|^{(1 / 2)}\right)^{N}\left(1+|x-y| \rho(x)^{-1}\right)^{N}} \\
& \times\left(\frac{1}{|x-y|^{n-2}} \int_{B(y,|x-y|)} \frac{V(z) d z}{|z-y|^{n-1}}+\frac{1}{|x-y|^{n-1}}\right) .
\end{aligned}
$$

By the functional calculus, we may write

$$
(-\Delta+V)^{-(1 / 2)}=\frac{1}{2 \pi} \int_{\mathbb{R}}(-i \tau)^{-(1 / 2)}(-\Delta+V+\tau)^{-1} d \tau .
$$

Let $f \in C_{0}^{\infty}\left(\mathbb{R}^{n}\right)$. From $(-\Delta+V+\tau)^{-1} f(x)=\int_{\mathbb{R}^{n}} \Gamma(x, y, \tau)$ $f(y) d y$, it follows that

$$
\mathscr{R} f(x)=\int_{\mathbb{R}^{n}} K(x, y) f(y) d y,
$$

where

$$
K(x, y)=\frac{1}{2 \pi} \int_{\mathbb{R}}(-i \tau)^{-(1 / 2)} \nabla_{x} \Gamma(x, y, \tau) d \tau .
$$

And the adjoint operator of $\mathscr{R}$ is given by

$$
\widetilde{\mathscr{R}} f(x)=\int_{\mathbb{R}^{n}} \widetilde{K}(x, y) f(y) d y,
$$

where

$$
\widetilde{K}(x, y)=\frac{1}{2 \pi} \overline{\int_{\mathbb{R}}(-i \tau)^{-(1 / 2)} \nabla_{y} \Gamma(y, x, \tau) d \tau} .
$$


Lemma 13. Suppose $V \in B_{s_{1}}$ for some $s_{1}>(n / 2)$. Then there exists $\delta>0$, for any integer $N>0$, and $0<h<(|x-y| / 16)$,

$$
\begin{aligned}
|K(x, y)| & \frac{C_{N}}{\left(1+|x-y| \rho(x)^{-1}\right)^{N}} \\
& \times\left(\frac{1}{|x-y|^{n-1}} \int_{B(y,|x-y|)} \frac{V(z) d z}{|z-y|^{n-1}}+\frac{1}{|x-y|^{n}}\right), \\
|K(x, y+h)-K(x, y)| & \frac{C_{N}}{\left(1+|x-y| \rho(x)^{-1}\right)^{N}} \frac{|h|^{\delta}}{|x-y|^{n-1+\delta}} \\
\quad & \quad\left(\int_{B(y,|x-y|)} \frac{V(z) d z}{|z-y|^{n-1}}+\frac{1}{|x-y|}\right) .
\end{aligned}
$$

Lemmas 12 and 13 have been proved in [9] and in [2], respectively.

Thirdly, we recall some important and useful properties of $A_{p}$ weights (cf. Chapter V in [13]).

Proposition 14. (i) Let $\omega \in A_{p}, 1 \leq p<\infty$. Then there exist a constant $C>0$ and $r>1$ depending only on $p$ and the $A_{p}$ constant of $\omega$, such that

$$
\left(\frac{1}{|B|} \int_{B} \omega^{r} d x\right)^{1 / r} \leq \frac{C}{|B|} \int_{B} \omega d x
$$

for any ball $B$.

(ii) Let $\omega \in A_{1}$. If $1 \leq p<\infty$, then $\omega^{1-p} \in A_{p}$.

At last, we review some basic facts about the BMO space $\mathrm{BMO}_{\theta}(\rho)$, which have been proved in [4].

Proposition 15. Let $\theta>0$ and let $1 \leq p<\infty$. If $b \in$ $\mathrm{BMO}_{\theta}(\rho)$, then

$$
\begin{gathered}
\left(\frac{1}{|B(x, r)|} \int_{B(x, r)}\left|b(y)-b_{B}\right|^{p} d y\right)^{(1 / p)} \\
\leq C[b]_{\theta}\left(1+\frac{r}{\rho(x)}\right)^{\theta^{\prime}}
\end{gathered}
$$

for all $B=B(x, r)$, with $x \in \mathbb{R}^{n}$ and $r>0$, where $\theta^{\prime}=\left(1+l_{0}\right) \theta$ and $l_{0}$ is the constant appearing in Lemma 9.

Lemma 16. Let $b \in B M O_{\theta}(\rho)$, let $B=B\left(x_{0}, r\right)$, and let $s \geq 1$; then

$$
\left(\frac{1}{\left|2^{k} B\right|} \int_{2^{k} B}\left|b(y)-b_{B}\right|^{p} d y\right)^{(1 / p)} \leq C[b]_{\theta} k\left(1+\frac{2^{k} r}{\rho\left(x_{0}\right)}\right)^{\theta^{\prime}},
$$

for all $k \in \mathbb{N}$, with $\theta^{\prime}$ as in (27).

\section{Weighted Estimates of Riesz Transforms Associated with the Schrödinger Operators}

In this section, we need to prove the weighted estimates of the Riesz transform associated with the Schrödinger operator, which will be used in the proof of Theorem 5 .

Theorem 17. Let $\omega \in A_{1}\left(\mathbb{R}^{n}\right)$. Suppose $V \in B_{\text {s }}$ for some $s \geq$ $(n / 2)$. Then, for $p_{0}^{\prime} \leq p<\infty$,

$$
\left\|(-\Delta+V)^{-(1 / 2)} \nabla f\right\|_{L^{p}(\omega)} \leq C\|f\|_{L^{p}(\omega)},
$$

where $p_{0}^{\prime}=p_{0} /\left(p_{0}-1\right)$ and $\left(1 / p_{0}\right)=(1 / s)-(1 / n)$.

Proof. By the improvement of " $B_{s}$ ", $V \in B_{s_{1}}$ for $s<s_{1}<n$. It follows from the proof of Theorem 0.5 in [9] that

$$
\begin{aligned}
\left|(-\Delta+V)^{1 / 2} \nabla f(x)\right| \leq & C\left\{M\left(|f|^{p_{1}^{\prime}}\right)(x)\right\}^{1 / p_{1}^{\prime}} \\
& +2 \sup _{\varepsilon>0}\left|\int_{|y-x|>\varepsilon} K_{1}^{0}(y, x) f(y) d y\right|,
\end{aligned}
$$

where $\left(1 / p_{1}^{\prime}\right)=1-\left(1 / p_{1}\right)=1-\left(1 / s_{1}\right)+(1 / n)$ and $K_{1}^{0}(x, y)$ is the kernel for the operator $\nabla(-\Delta)^{-1 / 2}$.

Since $\omega \in A_{1}\left(\mathbb{R}^{n}\right)$, then it follows from Proposition 7.2 in [14] that $\omega \in A_{p}\left(\mathbb{R}^{n}\right)$ for any $p>1$.

By [13, Theorem 1, page 201] and [13, Corollary 2, page 205]

$$
\left\|(-\Delta+V)^{-1 / 2} \nabla f\right\|_{L^{p}(\omega)} \leq C\|f\|_{L^{p}(\omega)} \text {, for } s_{1}^{\prime}<p<\infty \text {. }
$$

Since $p_{1}^{\prime}<p_{0}^{\prime}$, then

$$
\left\|(-\Delta+V)^{-1 / 2} \nabla f\right\|_{L^{p}(\omega)} \leq C\|f\|_{L^{p}(\omega)}, \quad \text { for } p_{0}^{\prime}<p<\infty \text {. }
$$

By duality, we have the following.

Corollary 18. Let $\omega \in A_{1}\left(\mathbb{R}^{n}\right)$. Suppose $V \in B_{s}$ for some $s \geq$ $(n / 2)$. Then, for $1<p \leq p_{0}$,

$$
\left\|\nabla(-\Delta+V)^{-1 / 2} f\right\|_{L^{p}(\omega)} \leq C\|f\|_{L^{p}(\omega)},
$$

where $\left(1 / p_{0}\right)=(1 / s)-(1 / n)$.

Proof. It follows from (ii) in Proposition 14 that $\omega^{1-p^{\prime}} \in A_{p^{\prime}}$ for $p_{0}^{\prime}<p^{\prime}<\infty$, where $(1 / p)+\left(1 / p^{\prime}\right)=1$. By using Theorem 17, we know that

$$
\left\|(-\Delta+V)^{-1 / 2} \nabla f\right\|_{L^{p^{\prime}\left(\omega^{1-p^{\prime}}\right)}} \leq C\|f\|_{L^{p^{\prime}}\left(\omega^{1-p^{\prime}}\right)} .
$$


Therefore, by duality and (34),

$$
\begin{aligned}
& \left\|\nabla(-\Delta+V)^{-1 / 2} f\right\|_{L^{p}(\omega)} \\
& =\sup _{\|g\|_{L^{p^{\prime}(\omega)}}=1}\left|\int_{\mathbb{R}^{n}} \nabla(-\Delta+V)^{-1 / 2} f(x) g(x) \omega(x) d x\right| \\
& =\sup _{\|g\|_{L^{p^{\prime}(\omega)}}=1}\left|\int_{\mathbb{R}^{n}} f(y)\left((-\Delta+V)^{-1 / 2} \nabla\right)(g \omega)(y) d y\right| \\
& \leq \sup _{\|g\|_{L^{p^{\prime}(\omega)}}=1}\left(\int_{\mathbb{R}^{n}}|f(y)|^{p} \omega(y) d y\right)^{1 / p} \\
& \times\left(\int_{\mathbb{R}^{n}}\left|\left((-\Delta+V)^{-1 / 2} \nabla\right)(g \omega)(y)\right|^{p^{\prime}}\right. \\
& \left.\times(\omega(y))^{-\left(p^{\prime} / p\right)} d y\right)^{1 / p^{\prime}} \\
& =\sup _{\|g\|_{L^{p^{\prime}}(\omega)}=1}\left(\int_{\mathbb{R}^{n}}|f(y)|^{p} \omega(y) d y\right)^{1 / p} \\
& \times\left(\int_{\mathbb{R}^{n}}\left|\left((-\Delta+V)^{-1 / 2} \nabla\right)(g \omega)(y)\right|^{p^{\prime}}\right. \\
& \left.\times(\omega(y))^{1-p^{\prime}} d y\right)^{1 / p^{\prime}} \\
& \leq C \sup _{\|g\|_{L^{p^{\prime}(\omega)}}=1}\left(\int_{\mathbb{R}^{n}}|f(y)|^{p} \omega(y) d y\right)^{1 / p} \\
& \times\left(\int_{\mathbb{R}^{n}}|g(y)|^{p^{\prime}} \omega(y) d y\right)^{1 / p^{\prime}} \\
& \leq C\|f\|_{L^{p}(\omega)} .
\end{aligned}
$$

This completes the proof of the above corollary.

In order to prove Theorem 4, we need the following lemmas.

Lemma 19 (see [11, Lemma 3.12]). Let $r=\rho(x)$. Then there exists a constant $C>0$, such that

$$
\int_{|x-y|>r}|K(y, x)| \omega(y) d y \leq C \omega(x) .
$$

By using the proof of Lemma 3.15 in [11], we immediately have the following.

Lemma 20. Let $r=\rho(x)$. Then there exists a constant $C>0$, such that

$$
\int_{|x-y| \leq r}\left|K(y, x)-K^{0}(y, x)\right| \omega(y) d y \leq C \omega(x),
$$

where $K^{0}(y, x)$ is the kernel of the operator $\nabla(-\Delta)^{-(1 / 2)}$.

Now we give the proof of Theorem 4 .
Proof of Theorem 4. We show Theorem 4 by a method similar to the one used in the proof of Theorem 2 in [15]. By the Calderón-Zygmund decomposition in the proof of Theorem 3.5 on page 413 in [16], given $f \in L^{1}(\omega)$ and $\alpha>0$, we have $f=f_{1}+f_{2}$, with $f_{2}=\sum_{k} b_{k}$, such that the following hold.

(1) $\left|f_{1}(x)\right| \leq C \alpha$, for a.e. $x \in \mathbb{R}^{n}$.

(2) Each $b_{k}$ is supported in a ball $B_{k}$,

$$
\int_{B_{k}}\left|b_{k}(x)\right| \omega(x) d x \leq C \alpha \omega\left(B_{k}\right), \quad \int_{B_{k}} b_{k}(x) d x=0 .
$$

(3) $\sum_{k} \omega\left(B_{k}\right) \leq(C / \alpha)\|f\|_{L^{1}(\omega)}$.

Because $\omega \in A_{1}\left(\mathbb{R}^{n}\right) \subseteq A_{2}\left(\mathbb{R}^{n}\right)$, by Corollary 18 , we know that $\mathscr{R}$ is bounded on $L^{2}(\omega)$; it is clear that

$$
\omega\left\{x \in \mathbb{R}^{n}:\left|\mathscr{R} f_{1}(x)\right|>\frac{\alpha}{2}\right\} \leq \frac{C}{\alpha^{2}}\left\|f_{1}\right\|_{L^{2}(\omega)}^{2} \leq \frac{C}{\alpha}\|f\|_{L^{1}(\omega)} .
$$

Let $B_{k}=B\left(x_{k}, r_{k}\right)$ and $\Omega=\bigcup_{k} B\left(x_{k}, 2 r_{k}\right)$. Then

$$
\omega(\Omega) \leq C \sum_{k} \omega\left(B_{k}\right) \leq \frac{C}{\alpha}\|f\|_{L^{1}(\omega)} .
$$

We only need to consider $\mathscr{R} f_{2}(x)$ for $x \in \Omega^{c}$. If $r_{k} \geq$ $\left(1 /\left(m\left(x_{k}, V\right)\right)\right)$, then $(1 /(m(y, V))) \leq C r_{k}$ for any $y \in B_{k}$. By Lemma 19, we get

$$
\begin{aligned}
& \int_{\left|x-x_{k}\right| \geq 2 r_{k}}\left|\mathscr{R} b_{k}(x)\right| d x \\
& \quad \leq \int_{\left|x-x_{k}\right| \geq 2 r_{k}} \int_{B_{k}}|K(x, y)|\left|b_{k}(y)\right| \omega(x) d y d x \\
& \leq C\left\|b_{k}\right\|_{L^{1}(\omega)} .
\end{aligned}
$$

If $r_{k}<1 /\left(m\left(x_{k}, V\right)\right)$, then $1 /\left(m\left(x_{k}, V\right)\right) \sim 1 /(m(y, V))$ for any $y \in B_{k}$. Since $K_{0}(x, y)$ is a Calderón-Zygmund kernel, by Lemma 19, Lemma 20, and [16, Lemma 3.3, page 413], we obtain

$$
\begin{aligned}
\int_{\left|x-x_{k}\right| \geq 2 r_{k}}\left|\mathscr{R} b_{k}(x)\right| \omega(x) d x \\
\leq \int_{2 r_{k} \leq\left|x-x_{k}\right|<2 /\left(m\left(x_{k}, V\right)\right)}\left|\mathscr{R} b_{k}(x)\right| \omega(x) d x \\
\quad+\int_{\left|x-x_{k}\right| \geq 2 /\left(m\left(x_{k}, V\right)\right)}\left|\mathscr{R} b_{k}(x)\right| \omega(x) d x
\end{aligned}
$$




$$
\begin{aligned}
\leq & \int_{2 r_{k} \leq\left|x-x_{k}\right|<2 /\left(m\left(x_{k}, V\right)\right)} \\
& \times \int_{B_{k}}\left|K(x, y)-K_{0}(x, y)\right|\left|b_{k}(y)\right| d y \omega(x) d x \\
& +\int_{2 r_{k} \leq\left|x-x_{k}\right|<2 /\left(m\left(x_{k}, V\right)\right)} \\
& \times \int_{B_{k}}\left|K_{0}(x, y)-K_{0}\left(x, x_{k}\right)\right|\left|b_{k}(y)\right| d y \omega(x) d x \\
& +\int_{\left|x-x_{k}\right| \geq 2 /\left(m\left(x_{k}, V\right)\right)} \int_{B_{k}}|K(x, y)|\left|b_{k}(y)\right| d y \omega(x) d x \\
\leq & C\left\|b_{k}\right\|_{L^{1}(\omega)} .
\end{aligned}
$$

Then

$$
\begin{gathered}
\int_{\Omega^{c}}\left|\mathscr{R} f_{2}(x)\right| d x \leq \sum_{k} \int_{\left(B\left(x, 2 r_{k}\right)\right)^{c}}\left|\mathscr{R} b_{k}(x)\right| \omega(x) d x \\
\leq C \sum_{k}\left\|b_{k}\right\|_{L^{1}(\omega)} \leq C \alpha \sum_{k} \omega\left(B_{k}\right) \leq C\|f\|_{L^{1}(\omega)^{\prime}} .
\end{gathered}
$$

Therefore

$$
\omega\left\{x \in \Omega^{c}:\left|\mathscr{R} f_{2}(x)\right|>\frac{\alpha}{2}\right\} \leq \frac{C}{\alpha}\|f\|_{L^{1}} .
$$
(44).

Theorem 4 is proved by combination of (39), (40), and

\section{Proof of Theorem 5}

For $f \in H_{L}^{1}(\omega)$ we can write $f=\sum_{j=-\infty}^{\infty} \lambda_{j} a_{j}$, where each $a_{j}$ is a $H_{L}^{1, \infty}(\omega)$ atom and $\sum_{j=-\infty}^{\infty}\left|\lambda_{j}\right| \leq 2\|f\|_{H_{L}^{1}(\omega)}$. Suppose that $\operatorname{supp} a_{j} \subseteq B_{j}=B\left(x_{j}, r_{j}\right)$ with $r_{j}<\rho\left(x_{j}\right)$. Write

$$
\begin{gathered}
\mathscr{R}_{b} f(x)=\sum_{j=-\infty}^{\infty} \lambda_{j}\left(b(x)-b_{B_{j}}\right) \mathscr{R} a_{j}(x) \chi_{8 B_{j}}(x) \\
+\sum_{j: r_{j} \geq\left(\rho\left(x_{j}\right) / 4\right)} \lambda_{j}\left(b(x)-b_{B_{j}}\right) \\
\times \mathscr{R} a_{j}(x) \chi_{\left(8 B_{j}\right)^{c}}(x) \\
+\sum_{j: r_{j}<\left(\rho\left(x_{j}\right) / 4\right)} \lambda_{j}\left(b(x)-b_{B_{j}}\right) \\
\quad \times \mathscr{R} a_{j}(x) \chi_{\left(8 B_{j}\right)^{c}}(x) \\
-\mathscr{R}\left(\sum_{j=-\infty}^{\infty} \lambda_{j}\left(b-b_{B_{j}}\right) a_{j}\right)(x) \\
=A_{1}(x)+A_{2}(x)+A_{3}(x)+A_{4}(x) .
\end{gathered}
$$

Using Hölder inequality, the weighted $L^{q}$ boundedness of $\mathscr{R}$, Propositions 14 and 15 , for $(1 / q)+(1 / t)+(1 / \nu)=1$ and $(1-(1 / q)) t=r$ with $1<q<p_{0}$, we have

$$
\begin{aligned}
\|(b(x) & \left.-b_{B}\right) \mathscr{R} a_{j}(x) \chi_{8 B_{j}}(x) \|_{L^{1}(\omega)} \\
\leq & \left(\int_{8 B_{j}}\left|b(x)-b_{B}\right|^{v} d x\right)^{1 / v} \\
& \times\left\|\mathscr{R} a_{j}\right\|_{L^{q}(\omega)}\left(\int_{8 B_{j}} \omega(x)^{r} d x\right)^{1 / t} \\
\leq & C\left|8 B_{j}\right|^{1 / v}\left|8 B_{j}\right|^{1 / t}\left(\frac{1}{\left|8 B_{j}\right|} \int_{8 B_{j}}\left|b(x)-b_{B}\right|^{\nu} d x\right)^{1 / v} \\
& \times\left\|a_{j}\right\|_{L^{q}(\omega)}\left(\frac{1}{\left|8 B_{j}\right|} \int_{8 B_{j}} \omega(x)^{r} d x\right)^{1 / t} \\
\leq & C[b]_{\theta}\left|8 B_{j}\right|^{1 / v}\left|8 B_{j}\right|^{1 / t} \omega\left(8 B_{j}\right)^{(1 / q)-1} \\
& \times\left(\frac{1}{\left|8 B_{j}\right|} \int_{8 B_{j}} \omega(x) d x\right)^{r / t} \\
\leq & C[b]_{\theta},
\end{aligned}
$$

since $r_{j}<\rho\left(x_{j}\right)$.

When we consider the term $A_{2}(x)$, we note that $\rho\left(x_{j}\right)>$ $r_{j} \geq \rho\left(x_{j}\right) / 4$ as following:

$$
\begin{aligned}
\|(b(x) & \left.-b_{B_{j}}\right) \mathscr{R} a_{j}(x) \chi_{\left(8 B_{j}\right)^{c}}(x) \|_{L^{1}(\omega)} \\
\lesssim & \int_{B_{j}}\left|a_{j}(y)\right| d y \\
& \times\left\{\int_{\left|x-x_{j}\right| \geq 8 r_{j}}|K(x, y)| b(x)-b_{B_{j}} \mid \omega(x) d x\right\} \\
\lesssim & \int_{B_{j}}\left|a_{j}(y)\right| d y \\
& \times\left\{\int_{\left|x-x_{j}\right| \geq 8 r_{j}} \frac{C_{l}}{(1+|x-y| / \rho(x))^{l}} \frac{1}{|x-y|^{n-1}}\right. \\
& \times\left(\int_{B\left(x, 2\left|x-x_{j}\right|\right)} \frac{V(z)}{|x-z|^{n-1}} d z+\frac{1}{|x-y|}\right) \\
& \times \int_{B_{j}}\left|a_{j}(y)\right|\left\{I_{1}(y)+I_{2}(y)\right\} d y .
\end{aligned}
$$

Note that $\left|x-x_{j}\right| \sim|x-y|$ and

$$
\begin{gathered}
\left(1+\frac{|x-y|}{\rho(x)}\right) \geq C\left(1+\frac{\left|x-x_{j}\right|}{\rho(x)}\right) \\
\geq C\left(1+\frac{\left|x-x_{j}\right|}{\rho\left(x_{j}\right)}\right)^{1 /\left(k_{0}+1\right)} .
\end{gathered}
$$


Then by Lemma 16 and Proposition 14, for any $y \in B_{j}$, $I_{2}(y)$

$$
\begin{gathered}
\leq \sum_{k=1}^{\infty} \int_{2^{k+3} r_{j} \leq\left|x-x_{j}\right|<2^{k+4} r_{j}} \frac{1}{\left(1+\left|x-x_{j}\right| \rho\left(x_{j}\right)^{-1}\right)^{N /\left(k_{0}+1\right)}} \\
\times \frac{1}{\left|x-x_{j}\right|^{n}}\left|b(x)-b_{B_{j}}\right| \omega(x) d x
\end{gathered}
$$$$
\lesssim \sum_{k=1}^{\infty} 2^{-((k+1) N) /\left(k_{0}+1\right)} \frac{1}{\left|B\left(x, 2^{k+3} r_{j}\right)\right|}
$$$$
\times \int_{\left|x-x_{j}\right|<2^{k+4} r_{j}}\left|b-b_{B_{j}}\right| \omega(x) d x
$$$$
\lesssim \sum_{k=1}^{\infty} 2^{-\left(((k+1) N) /\left(k_{0}+1\right)\right)}\left(\frac{1}{\left|2^{k+4} B_{j}\right|} \int_{2^{k+4} B_{j}}\left|b-b_{B_{j}}\right|^{r^{\prime}} d x\right)^{1 / r^{\prime}}
$$$$
\times\left(\frac{1}{\left|2^{k+4} B_{j}\right|} \int_{2^{k+4} B_{j}} \omega(x)^{r} d x\right)^{1 / r}
$$$$
\lesssim \sum_{k=1}^{\infty} 2^{-((k+1) N) /\left(k_{0}+1\right)}[b]_{\theta} k\left(1+\frac{2^{k+4} r_{j}}{\rho\left(x_{j}\right)}\right)^{\left(k_{0}+1\right) \theta}
$$$$
\times\left(\frac{1}{\left|2^{k+4} B_{j}\right|} \int_{2^{k+4} B_{j}} \omega(x) d x\right)
$$$$
\lesssim \sum_{k=1}^{\infty} 2^{-((k+1) N) /\left(k_{0}+1\right)}[b]_{\theta} k\left(1+\frac{2^{k+4} r_{j}}{\rho\left(x_{j}\right)}\right)^{\left(k_{0}+1\right) \theta}
$$$$
\times\left(\frac{1}{\left|2^{k+5} B_{j}\right|} \int_{B\left(y, 2^{k+5} r_{j}\right)} \omega(x) d x\right)
$$$$
\lesssim \sum_{k=1}^{\infty} 2^{-((k+1) N) /\left(k_{0}+1\right)}[b]_{\theta} k\left(1+\frac{2^{k+4} r_{j}}{\rho\left(x_{j}\right)}\right)^{\left(k_{0}+1\right) \theta} M(w)(y)
$$$$
\leq C[b]_{\theta} M(\omega)(y),
$$

where we have chosen $N$ large enough.

Similarly, for $(1 / p)+(1 / q)+(1 / r)=1$ and $\left(1 / s_{1}\right)=$ $(1 / p)+(1 / n)$, using Hölder inequality, Proposition 14 , and the boundedness of the fractional integral $\mathscr{I}_{1}$, we obtain, for any $y \in B_{j}$,

$$
\begin{aligned}
I_{1}(y) \lesssim & \sum_{k=1}^{\infty} \int_{2^{k+3} r_{j} \leq\left|x-x_{j}\right|<2^{k+4} r_{j}} \frac{1}{\left(1+\left|x-x_{j}\right| \rho\left(x_{j}\right)^{-1}\right)^{N /\left(k_{0}+1\right)}} \\
& \times \frac{\left|b(x)-b_{B_{j}}\right|}{\left|x-x_{j}\right|^{n-1}} \mathscr{I}_{1}\left(V \chi_{2^{k+4} B_{j}}\right)(x) \omega(x) d x
\end{aligned}
$$

$$
\begin{aligned}
& \lesssim \sum_{k=1}^{\infty} 2^{-((k+1) N) /\left(k_{0}+1\right)} \frac{1}{\left(2^{k+3} r_{j}\right)^{n-1}} \\
& \times \int_{\left|x-x_{j}\right|<2^{k+4} r_{j}} \mathcal{I}_{1}\left(V \chi_{2^{k+4} B_{j}}\right)(x)\left|b-b_{B_{j}}\right| \omega(x) d x \\
& \leq \sum_{k=1}^{\infty} 2^{-((k+1) N) /\left(k_{0}+1\right)}\left(2^{k+3} r_{j}\right)^{1-n+(n / q)+(n / r)} \\
& \times\left(\frac{1}{\left(2^{k+3} r_{j}\right)^{n}} \int_{\left|x-x_{j}\right|<2^{k+4} r_{j}}\left|b-b_{B_{j}}\right|^{q} d x\right)^{1 / q} \\
& \quad \times\left(\frac{1}{\left|2^{k+3} B_{j}\right|} \int_{2^{k+3} B_{j}} \omega(x)^{r} d x\right)^{1 / r} \\
& \times\left\|\mathscr{I}_{1}\left(V \chi_{2^{k+4} B_{j}}\right)\right\|_{p}
\end{aligned}
$$$$
\lesssim \sum_{k=1}^{\infty} 2^{-((k+1) N) /\left(k_{0}+1\right)}\left(2^{k+3} r_{j}\right)^{2-\left(n / s_{1}\right)}
$$$$
\times\left(\frac{1}{\left(2^{k+3} r_{j}\right)^{n}} \int_{\left|x-x_{j}\right|<2^{k+4} r_{j}}\left|b-b_{B_{j}}\right|^{q} d x\right)^{1 / q}
$$$$
\times\left(\frac{1}{\left|2^{k+3} B_{j}\right|} \int_{2^{k+3} B_{j}} \omega(x) d x\right)\left\|V \chi_{2^{k+4} B_{j}}\right\|_{s_{1}}
$$$$
\lesssim \sum_{k=1}^{\infty} 2^{-((k+1) N) /\left(k_{0}+1\right)}[b]_{\theta} k\left(1+\frac{2^{k+4} r_{j}}{\rho\left(x_{j}\right)}\right)^{\left(k_{0}+1\right) \theta}
$$$$
\times\left(\frac{1}{\left|2^{k+5} B_{j}\right|} \int_{B\left(y, 2^{k+5} r_{j}\right)} \omega(x) d x\right)
$$$$
\times\left(2^{k+3} r_{j}\right)^{2-n / s_{1}}\left\|V \chi_{2^{k+4} B_{j}}\right\|_{s_{1}}
$$$$
\lesssim \sum_{k=1}^{\infty} 2^{-((k+1) N) /\left(k_{0}+1\right)}[b]_{\theta} k\left(1+\frac{2^{k+4} r_{j}}{\rho\left(x_{j}\right)}\right)^{\left(k_{0}+1\right) \theta}
$$$$
\times M(\omega)(y) \frac{1}{\left(2^{k+3} r_{j}\right)^{n-2}} \int_{2^{k+3} B_{j}} V d x
$$$$
\lesssim \sum_{k=1}^{\infty} 2^{-((k+1) N) /\left(k_{0}+1\right)}[b]_{\theta} k\left(1+\frac{2^{k+4} r_{j}}{\rho\left(x_{j}\right)}\right)^{\left(k_{0}+1\right) \theta}
$$$$
\times\left(1+\frac{2^{k+4} r_{j}}{\rho\left(x_{j}\right)}\right)^{l_{1}} M(\omega)(y)
$$$$
\leq C[b]_{\theta} M(\omega)(y),
$$

where we have chosen $N$ large enough and we have used Lemma 10 in the sixth inequality above. 
Therefore,

$$
\begin{aligned}
\int_{B_{j}}\left|a_{j}(y)\right|\left\{I_{1}(y)+I_{2}(y)\right\} d y \\
\quad \leq C[b]_{\theta} \int_{B_{j}}\left|a_{j}(y)\right| M(\omega)(y) d y \\
\quad \leq C[b]_{\theta} \int_{B_{j}}\left|a_{j}(y)\right| \omega(y) d y \leq C[b]_{\theta},
\end{aligned}
$$

where we have used the fact that $\omega \in A_{1}$.

Thus, if $\rho\left(x_{j}\right)>r_{j} \geq\left(\rho\left(x_{j}\right)\right) / 4$, then

$$
\left\|\left(b(x)-b_{B_{j}}\right) \mathscr{R} a_{j}(x) \chi_{\left(8 B_{j}\right)^{c}}(x)\right\|_{L^{1}(\omega)} \leq C[b]_{\theta} .
$$

For $A_{3}$, by using the vanishing condition of $a_{j}$ and Lemma 13, then

$$
\begin{aligned}
& \|(b(x)\left.-b_{B_{j}}\right)\left.\mathscr{R} a_{j}(x) \chi_{\left(8 B_{j}\right)^{c}}(x)\right|_{L^{1}(\omega)} \\
& \leq \int_{B_{j}}\left|a_{j}(y)\right| d y \\
& \times\left\{\int_{\left|x-x_{j}\right| \geq 8 r_{j}}\left|K(x, y)-K\left(x, x_{j}\right)\right|\right. \\
&\left.\times\left|b(x)-b_{B_{j}}\right| \omega(x) d x\right\} \\
& \lesssim \int_{B_{j}}\left|a_{j}(y)\right| d y \\
& \times\left\{\int_{\left|x-x_{j}\right| \geq 8 r_{j}} \frac{C_{l}}{(1+|x-y| / \rho(x))^{N}} \frac{\left|y-x_{j}\right|^{\delta}}{|x-y|^{n-1+\delta}}\right. \\
& \quad \times\left(\int_{B(x, 2|x-y|)} \frac{|V(z)|}{|x-z|^{n-1}} d z+\frac{1}{|x-y|}\right) \\
&\left.\quad \times\left|b(x)-b_{B_{j} \mid}\right| \omega(x) d x\right\} \\
& \quad \int_{B_{j}}\left|a_{j}(y)\right|\left\{\widetilde{I}_{1}(y)+\widetilde{I}_{2}(y)\right\} d y .
\end{aligned}
$$

First of all, we need to obtain the following estimate:

$$
\begin{aligned}
\left\|V \chi_{B\left(x_{j}, 2^{k+3} r_{j}\right)}\right\|_{s_{1}} \lesssim & \left(2^{k} r_{j}\right)^{-\left(n / s_{1}^{\prime}\right)} \int_{B\left(x_{j}, 2^{k} r_{j}\right)} V(z) d z \\
\lesssim & \left(2^{k} r_{j}\right)^{n-2-\left(n / s_{1}^{\prime}\right)} \frac{1}{\left(2^{k} r_{j}\right)^{n-2}} \\
& \times \int_{B\left(x_{j}, 2^{k} r_{j}\right)} V(z) d z \\
& \leqslant\left(2^{k} r_{j}\right)^{n-2-\left(n / s_{1}^{\prime}\right)}\left(1+2^{k} r_{j} m\left(x_{j},|V|\right)\right)^{l_{1}},
\end{aligned}
$$

where we have used Lemma 10 in the last inequality above.
Similarly, for $(1 / p)+(1 / q)+(1 / r)=1$ and $\left(1 / s_{1}\right)=$ $(1 / p)+(1 / n)$, using Hölder inequality, Proposition 14, and the boundedness of the fractional integral $\mathscr{I}_{1}$, we obtain, for any $y \in B_{j}$,

$$
\begin{aligned}
& \widetilde{I}_{1}(y) \lesssim \sum_{k=1}^{\infty} \int_{2^{k+3} r_{j} \leq\left|x-x_{j}\right|<2^{k+4} r_{j}} \frac{\mathscr{I}_{1}\left(V \chi_{2^{k+4} B_{j}}\right)(x)}{\left(1+\left|x-x_{j}\right| \rho\left(x_{j}\right)^{-1}\right)^{N /\left(l_{0}+1\right)}} \\
& \times \frac{\left|x_{j}-y\right|^{\delta}\left|b(x)-b_{B_{j}}\right|}{\left|x-x_{j}\right|^{n-1}\left|x-x_{j}\right|^{\delta}} \omega(x) d x \\
& \lesssim \sum_{k=1}^{\infty} 2^{-(k+3) \delta} \frac{\left(2^{k+3} r_{j}\right)^{1-n+n / q+n / r}}{\left(1+2^{k} r_{j} \rho\left(x_{j}\right)^{-1}\right)^{N /\left(l_{0}+1\right)}} \\
& \times\left(\frac{1}{\left|B\left(x, 2^{k+3} r_{j}\right)\right|} \int_{\left|x-x_{j}\right|<2^{k+4} r_{j}}\left|b-b_{B_{j}}\right|^{q} d x\right)^{1 / q} \\
& \times\left(\frac{1}{\left|B\left(x, 2^{k+3} r_{j}\right)\right|} \int_{\left|x-x_{j}\right|<2^{k+4} r_{j}} \omega(x)^{r} d x\right)^{1 / r} \\
& \times\left\|\mathscr{I}_{1}\left(V \chi_{2^{k+4} B_{j}}\right)\right\|_{p} \\
& \lesssim \sum_{k=1}^{\infty} 2^{-(k+3) \delta} \frac{\left(2^{k+3} r_{j}\right)^{1-n / p}}{\left(1+2^{k} r_{j} \rho\left(x_{j}\right)^{-1}\right)^{N /\left(l_{0}+1\right)}} \\
& \times\left(\frac{1}{\left|B\left(x, 2^{k+3} r_{j}\right)\right|} \int_{\left|x-x_{j}\right|<2^{k+4} r_{j}}\left|b-b_{B_{j}}\right|^{q} d x\right)^{1 / q} \\
& \times\left(\frac{1}{\left|B\left(x, 2^{k+3} r_{j}\right)\right|} \int_{\left|x-x_{j}\right|<2^{k+4} r_{j}} \omega(x)^{r} d x\right)^{1 / r} \\
& \times\left\|V \chi_{2^{k+4} B_{j}}\right\|_{s_{1}} \\
& \lesssim \sum_{k=1}^{\infty} 2^{-(k+3) \delta} \frac{\left(2^{k+3} r_{j}\right)^{2-n / s_{1}}}{\left(1+2^{k} r_{j} \rho\left(x_{j}\right)^{-1}\right)^{N /\left(l_{0}+1\right)}} \\
& \times\left(\frac{1}{\left|B\left(x, 2^{k+3} r_{j}\right)\right|} \int_{\left|x-x_{j}\right|<2^{k+4} r_{j}}\left|b-b_{B_{j}}\right|^{q} d x\right)^{1 / q} \\
& \times\left(\frac{1}{\left|B\left(x, 2^{k+3} r_{j}\right)\right|} \int_{\left|x-x_{j}\right|<2^{k+4} r_{j}} \omega(x) d x\right) \\
& \times\left\|V \chi_{2^{k+4} B_{j}}\right\|_{s_{1}} \\
& \lesssim \sum_{k=1}^{\infty} 2^{-(k+3) \delta} \frac{\left(2^{k+3} r_{j}\right)^{2}}{\left(1+2^{k} r_{j} \rho\left(x_{j}\right)^{-1}\right)^{N /\left(l_{0}+1\right)}}
\end{aligned}
$$




$$
\begin{aligned}
& \quad \times\left(\frac{1}{\left|B\left(x, 2^{k+3} r_{j}\right)\right|} \int_{\left|x-x_{j}\right|<2^{k+4} r_{j}}\left|b-b_{B_{j}}\right|^{q} d x\right)^{1 / q} \\
& \quad \times\left(\frac{1}{\left|B\left(x, 2^{k+3} r_{j}\right)\right|} \int_{\left|x-x_{j}\right|<2^{k+4} r_{j}} \omega(x) d x\right) \\
& \quad \times\left(\frac{1}{\left|B\left(x, 2^{k+3} r_{j}\right)\right|} \int_{\left|x-x_{j}\right|<2^{k+4} r_{j}} V(z) d z\right) \\
& \leqslant \sum_{k=1}^{\infty} 2^{-(k+3) \delta} \frac{\left(2^{k+3} r_{j}\right)^{2}}{\left(1+2^{k} r_{j} \rho\left(x_{j}\right)^{-1}\right)^{N /\left(l_{0}+1\right)}} \\
& \quad \times\left(\frac{1}{\left|B\left(x, 2^{k+3} r_{j}\right)\right|} \int_{\left|x-x_{j}\right|<2^{k+4} r_{j}}\left|b-b_{B_{j}}\right|^{q} d x\right)^{1 / q} \\
& \quad \times\left(\frac{1}{\left|B\left(y, 2^{k+5} r_{j}\right)\right|} \int_{|x-y|<2^{k+5} r_{j}} \omega(x) d x\right) \\
& \quad \times\left(\frac{1}{\left|B\left(x, 2^{k+3} r_{j}\right)\right|} \int_{\left|x-x_{j}\right|<2^{k+4} r_{j}} V(z) d z\right) \\
& \leqslant \sum_{k=1}^{\infty} 2^{-(k+3) \delta}[b]_{\theta} k\left(1+\frac{2^{k+4} r_{j}}{\rho\left(x_{j}\right)}\right)^{\left(k_{0}+1\right) \theta} M(\omega)(y) \\
& \leqslant \sum_{k=1}^{\infty} 2^{-(k+3) \delta}[b]_{\theta} k M(\omega)(y) \\
& \leqslant[b]_{\theta} M(\omega)(y), \\
& \quad \times\left(1+2^{k} r_{j} m\left(x_{j}, V\right)\right)^{\left.l_{1}-\left(N / l_{0}+1\right)\right)}
\end{aligned}
$$

where we have chosen $N$ large enough.

Secondly, for any $y \in B_{j}$,

$$
\begin{aligned}
& \widetilde{I}_{2}(y) \\
& \leq \int_{B_{j}}\left|a_{j}(y)\right| d y \\
& \quad \times \sum_{k=1}^{\infty} \int_{2^{k+3} r_{j} \leq\left|x-x_{j}\right|<2^{k+4} r_{j}} \frac{C_{l}}{\left(1+\left|x-x_{j}\right| \rho\left(x_{j}\right)^{-1}\right)^{N /\left(l_{0}+1\right)}} \\
& \quad \times \frac{\left|x_{j}-y\right|^{\delta}\left|b(x)-b_{B_{j}}\right|}{\left|x-x_{j}\right|^{n}\left|x-x_{j}\right|^{\delta}} \omega(x) d x \\
& \quad \sum_{k=1}^{\infty} 2^{-(k+3) \delta}\left(1+\frac{2^{k+3} r_{j}}{\rho\left(x_{j}\right)}\right)^{-N /\left(l_{0}+1\right)} \\
& \quad \times \int_{\left|x-x_{j}\right|<2^{k+4} r_{j}}\left|b-b_{B_{j}}\right| \omega(x) d x \\
& \quad \sum_{k=1}^{\infty} 2^{-(k+3) \delta}\left(1+\frac{2^{k+3} r_{j}}{\rho\left(x_{j}\right)}\right)^{-N /\left(l_{0}+1\right)}
\end{aligned}
$$

$$
\begin{aligned}
& \times\left(\frac{1}{\left|2^{k+4} B_{j}\right|} \int_{2^{k+4} B_{j}}\left|b-b_{B_{j}}\right|^{r^{\prime}} d x\right)^{1 / r^{\prime}} \\
& \times\left(\frac{1}{\left|2^{k+4} B_{j}\right|} \int_{2^{k+4} B_{j}} \omega(x)^{r} d x\right)^{1 / r} \\
& \leqslant \sum_{k=1}^{\infty} 2^{-(k+3) \delta}[b]_{\theta} k\left(1+\frac{2^{k+4} r_{j}}{\rho\left(x_{j}\right)}\right)^{\left(k_{0}+1\right) \theta-\left(N /\left(l_{0}+1\right)\right)} \\
& \times\left(\frac{1}{\left|2^{k+4} B_{j}\right|} \int_{2^{k+4} B_{j}} \omega(x) d x\right) \\
& \leqslant \sum_{k=1}^{\infty} 2^{-(k+3) \delta}[b]_{\theta} k\left(\frac{1}{\left|2^{k+5} B_{j}\right|} \int_{B\left(y, 2^{k+5} r_{j}\right)} \omega(x) d x\right) \\
& \leqslant \sum_{k=1}^{\infty} 2^{-(k+3) \delta}[b]_{\theta} k M(\omega)(y) \\
& \leqslant[b]_{\theta} M(\omega)(y),
\end{aligned}
$$

where we have also chosen $N$ large enough.

Therefore,

$$
\begin{gathered}
\int_{B_{j}}\left|a_{j}(y)\right|\left\{\widetilde{I}_{1}(y)+\widetilde{I}_{2}(y)\right\} d y \\
\leq C[b]_{\theta} \int_{B_{j}}\left|a_{j}(y)\right| M(\omega)(y) d y \\
\leq C[b]_{\theta} \int_{B_{j}}\left|a_{j}(y)\right| \omega(y) d y \leq C[b]_{\theta} .
\end{gathered}
$$

Therefore, if $r_{j} \leq \rho\left(x_{j}\right) / 4$, then

$$
\left\|\left(b(x)-b_{B_{j}}\right) \mathscr{R} a_{j}(x) \chi_{\left(8 B_{j}\right)^{c}}(x)\right\|_{L^{1}(\omega)} \leq C[b]_{\theta} .
$$

Thus, we have

$$
\begin{gathered}
\omega\left\{x \in \mathbb{R}^{n}:\left|A_{i}(x)\right|>\frac{\lambda}{4}\right\} \leq \frac{C}{\lambda}\left\|A_{i}(x)\right\|_{L^{1}(\omega)} \\
\leq \frac{C[b]_{\theta}}{\lambda} \sum_{j=-\infty}^{\infty}\left|\lambda_{j}\right|, \quad i=1,2,3 .
\end{gathered}
$$

Note that, for $\left(1 / r^{\prime}\right)+(1 / r)=1$,

$$
\begin{aligned}
& \left\|\left(b-b_{B_{j}}\right) a_{j}\right\|_{L^{1}(\omega)} \\
& \leq\left|8 B_{j}\right|\left(\frac{1}{\left|8 B_{j}\right|} \int_{8 B_{j}}\left|b(x)-b_{B}\right|^{r^{\prime}} d x\right)^{1 / r^{\prime}}\left\|a_{j}\right\|_{L^{\infty}(\omega)} \\
& \quad \times\left(\frac{1}{\left|8 B_{j}\right|} \int_{8 B_{j}} \omega(x)^{r} d x\right)^{1 / r}
\end{aligned}
$$




$$
\begin{aligned}
& \leq\left|8 B_{j}\right|\left(\frac{1}{\left|8 B_{j}\right|} \int_{B_{j}}\left|b(x)-b_{B}\right|^{r^{\prime}} d x\right)^{1 / r^{\prime}} \omega\left(B_{j}\right)^{-1} \\
& \times\left(\frac{1}{\left|8 B_{j}\right|} \int_{8 B_{j}} \omega(x) d x\right) \\
& \leq C[b]_{\theta}\left(1+\frac{r_{j}}{\rho\left(x_{j}\right)}\right)^{\theta^{\prime}} \\
& \leq C[b]_{\theta},
\end{aligned}
$$

where $r_{j}<\rho\left(x_{j}\right)$.

By Theorem 4, we get

$$
\begin{aligned}
\omega\{x & \left.\in \mathbb{R}^{n}:\left|A_{4}(x)\right|>\frac{\lambda}{4}\right\} \leq \frac{C}{\lambda} \| \sum_{j=-\infty}^{\infty} \lambda_{j}\left(b-b\left(x_{j}\right) a_{j} \|_{L^{1}(\omega)}\right. \\
& \leq \frac{C[b]_{\theta}}{\lambda} \sum_{j=-\infty}^{\infty}\left|\lambda_{j}\right| .
\end{aligned}
$$

Therefore,

$$
\begin{aligned}
\omega\{x & \left.\in \mathbb{R}^{n}:|[b, \mathscr{R}] f(x)|>\frac{\lambda}{4}\right\} \\
& \leq C \sum_{i=1}^{4} \omega\left\{x \in \mathbb{R}^{n}:\left|A_{i}(x)\right|>\frac{\lambda}{4}\right\} \\
& \leq \frac{C[b]_{\theta}}{\lambda} \sum_{j=-\infty}^{\infty}\left|\lambda_{j}\right| \\
& \leq \frac{C[b]_{\theta}}{\lambda}\|f\|_{H_{L}^{1}(\omega)} .
\end{aligned}
$$

This completes the proof of Theorem 5 .

\section{References}

[1] R. R. Coifman, R. Rochberg, and G. Weiss, "Factorization theorems for Hardy spaces in several variables," Annals of Mathematics, vol. 103, no. 3, pp. 611-635, 1976.

[2] Z. Guo, P. Li, and L. Peng, " $L_{p}$ boundedness of commutators of Riesz transforms associated to Schrödinger operator," Journal of Mathematical Analysis and Applications, vol. 341, no. 1, pp. 421432, 2008.

[3] P. Li and L. Peng, "Endpoint estimates for commutators of Riesz transforms associated with Schrödinger operators," Bulletin of the Australian Mathematical Society, vol. 82, no. 3, pp. 367-389, 2010.

[4] B. Bongioanni, E. Harboure, and O. Salinas, "Commutators of Riesz transforms related to Schrödinger operators," The Journal of Fourier Analysis and Applications, vol. 17, no. 1, pp. 115-134, 2011.

[5] B. Bongioanni, E. Harboure, and O. Salinas, "Weighted inequalities for commutators of Schrödinger-Riesz transforms," Journal of Mathematical Analysis and Applications, vol. 392, no. 1, pp. 622, 2012.

[6] Y. Liu, "Commutators of BMO functions and degenerate Schrödinger operators with certain nonnegative potentials," Monatshefte für Mathematik, vol. 165, no. 1, pp. 41-56, 2012.

[7] Y. Liu, J. Z. Huang, and J. F. Dong, "Commutators of higher order Riesz transform associated with Schrödinger operators," Journal of Function Spaces and Applications, vol. 2013, Article ID 842375, 15 pages, 2013.

[8] Y. Liu, J. Z. Huang, and J. F. Dong, “Commutators of CalderónZygmund operators related to admissible functions on spaces of homogeneous type and applications to Schrödinger operators," Science China Mathematics, vol. 56, no. 9, pp. 1895-1913, 2013.

[9] Z. W. Shen, " $L_{p}$ estimates for Schrödinger operators with certain potentials," Annales de l'Institut Fourier, vol. 45, no. 2, pp. 513-546, 1995.

[10] J. Dziubański and J. Zienkiewicz, "Hardy space $H^{1}$ associated to Schrödinger operator with potential satisfying reverse Hölder inequality," Revista Matemática Iberoamericana, vol. 15, no. 2, pp. 279-296, 1999.

[11] H. Liu, L. Tang, and H. Zhu, "Weighted Hardy spaces and BMO spaces associated with Schrödinger operators," Mathematische Nachrichten, vol. 285, no. 17-18, pp. 2173-2207, 2012.

[12] D. Yang and Y. Zhou, "Localized Hardy spaces $H^{1}$ related to admissible functions on $\mathrm{RD}$-spaces and applications to Schrödinger operators," Transactions of the American Mathematical Society, vol. 363, no. 3, pp. 1197-1239, 2011.

[13] E. M. Stein, Real-Variable Methods, Orthogonality, and Oscillatory Integrals, vol. 43 of Princeton Mathematical Series, Princeton University Press, Princeton, NJ, USA, 1993.

[14] J. Duoandikoetxea, Fourier Analysis, vol. 29 of Graduate Studies in Mathematics, American Mathematical Society, Providence, RI, USA, 2001.

[15] C. C. Lin, H. P. Liu, and Y. Liu, "Hardy spaces associated with Schrödinger operators on theHeisenberg group," http://arxiv.org/abs/1106.4960.

[16] J. García-Cuerva and J. L. Rubio de Francia, Weighted Norm Inequalities and Related Topics, vol. 116 of North-Holland Mathematics Studies, North-Holland, Amsterdam, The Netherlands, 1985. 


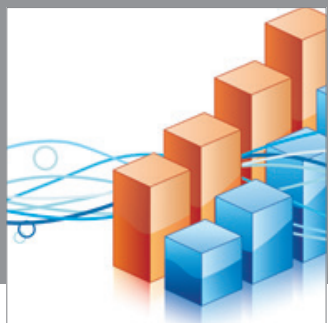

Advances in

Operations Research

mansans

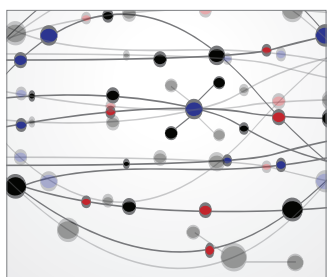

The Scientific World Journal
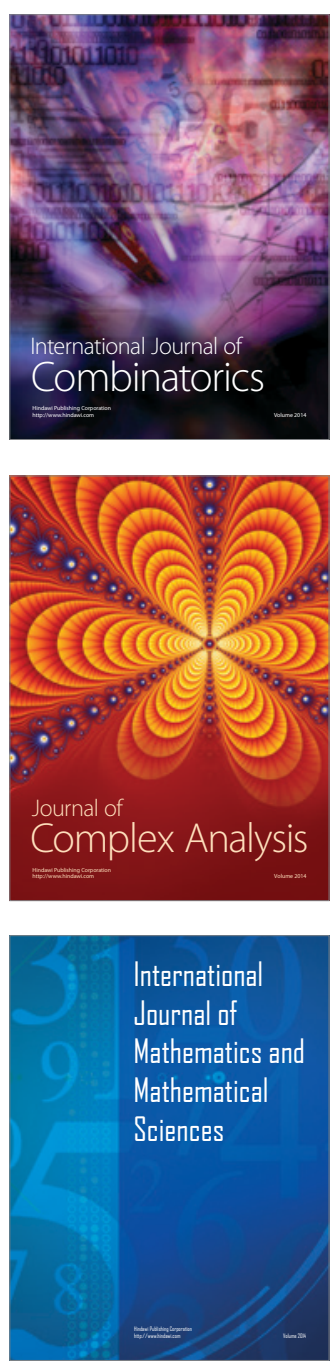
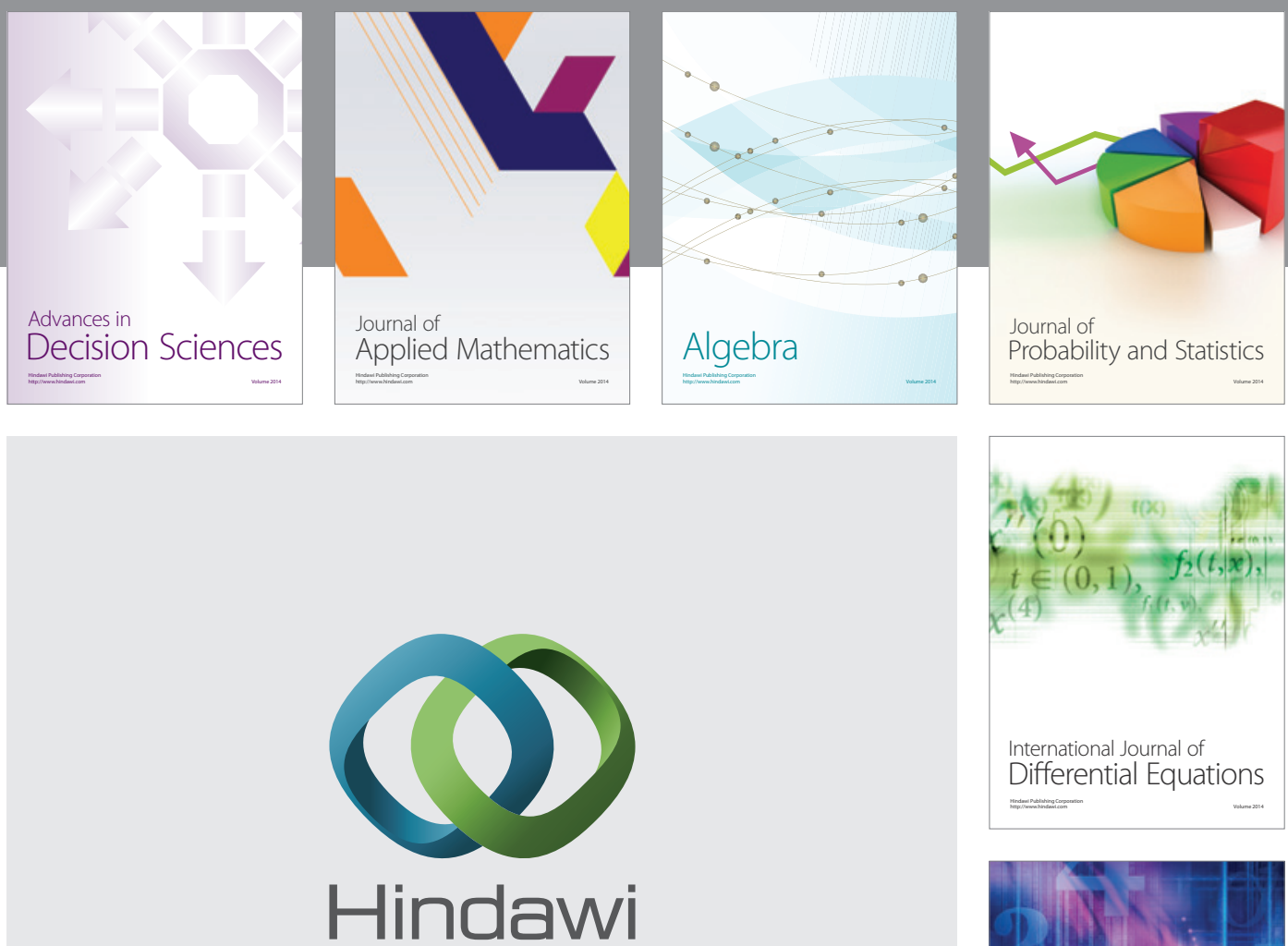

Submit your manuscripts at http://www.hindawi.com
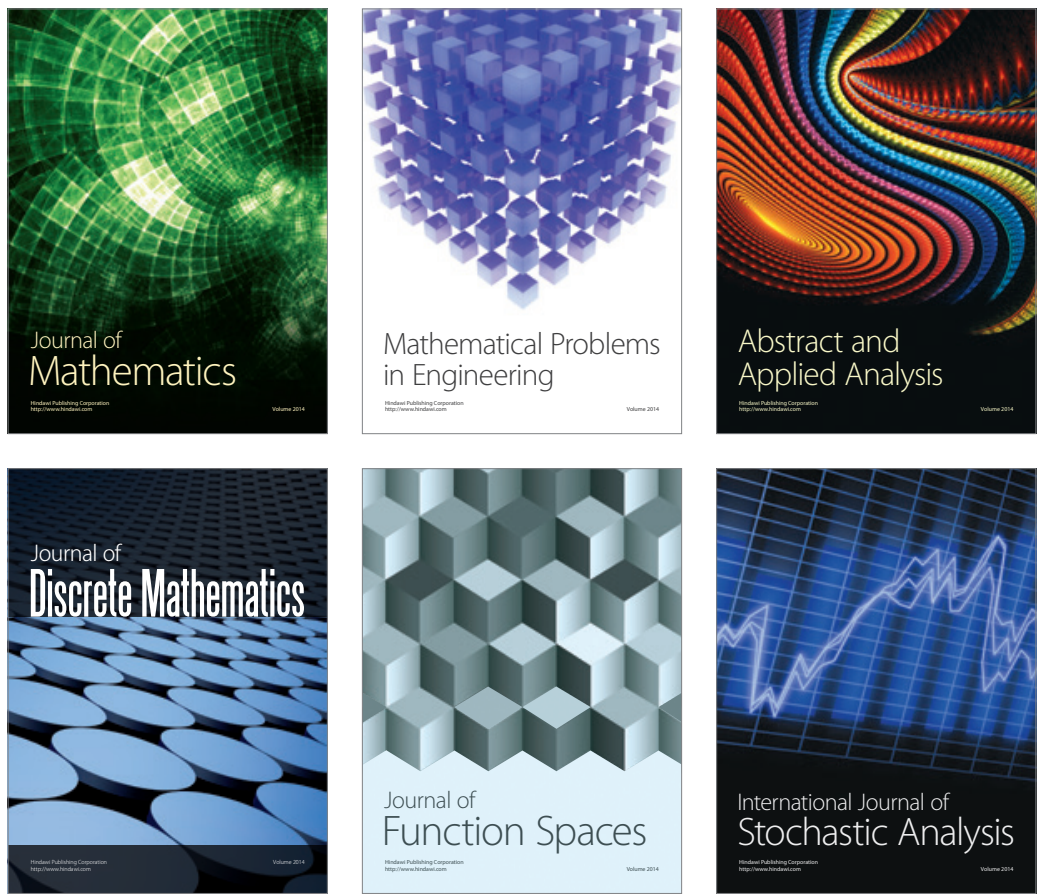

Journal of

Function Spaces

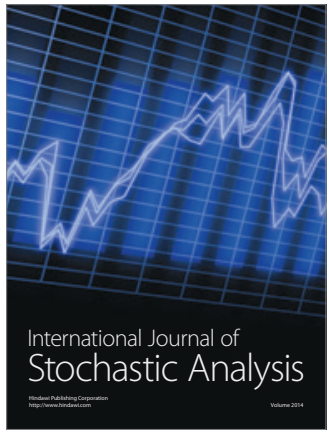

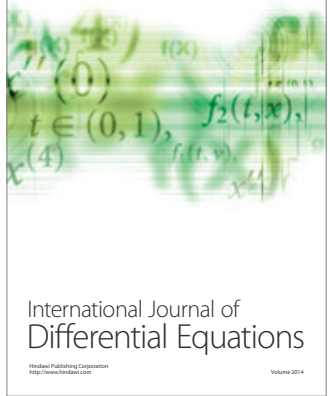
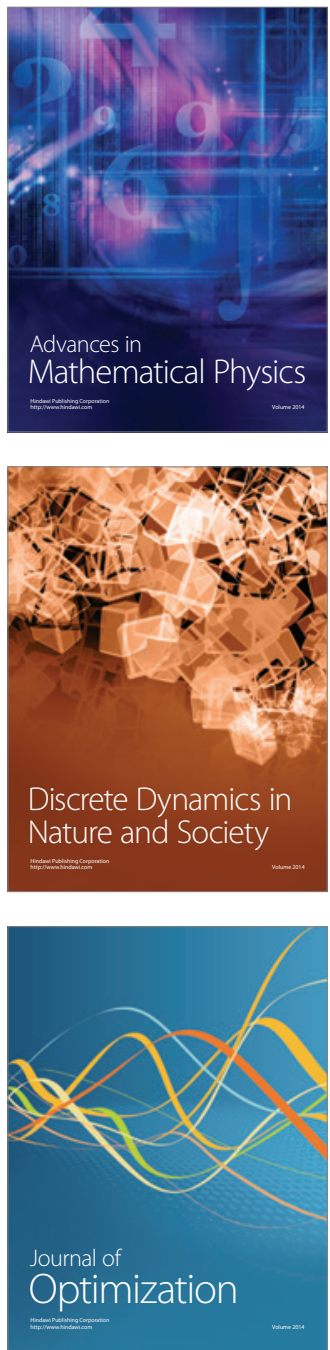